\title{
Generalized adjoint forms on algebraic varieties
}

\author{
Luca Rizzi $^{1}$ (D) Francesco Zucconi ${ }^{1}$
}

Received: 22 September 2015 / Accepted: 25 July 2016 / Published online: 10 August 2016

(C) Fondazione Annali di Matematica Pura ed Applicata and Springer-Verlag Berlin Heidelberg 2016

\begin{abstract}
We prove a full generalization of the Castelnuovo's free pencil trick. We show its analogies with Rizzi and Zucconi (Differential forms and quadrics of the canonical image. arXiv:1409.1826, Theorem 2.1.7); see also Pirola and Zucconi (J Algebraic Geom 12(3):535572, Theorem 1.5.1). Moreover we find a new formulation of the Griffiths's infinitesimal Torelli Theorem for smooth projective hypersurfaces using meromorphic 1-forms.
\end{abstract}

Keywords Extension class of a vector bundle · Torsion freeness · Castelnuovo's free pencil trick · Infinitesimal Torelli problem $\cdot$ Projective hypersurface $\cdot$ Meromorphic forms

Mathematics Subject Classification $14 \mathrm{C} 34 \cdot 14 \mathrm{D} 07 \cdot 14 \mathrm{~J} 10 \cdot 14 \mathrm{~J} 40 \cdot 14 \mathrm{~J} 70$

\section{Contents}

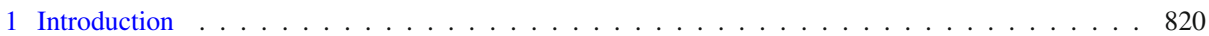

2 The theory of generalized adjoint forms . . . . . . . . . . . . . . . . . . . . . . 821

2.1 Definition of generalized adjoint form . . . . . . . . . . . . . . . . . . 821

2.2 Castelnuovo's free pencil trick . . . . . . . . . . . . . . . . . . . . . . 823

2.3 The Adjoint Theorem . . . . . . . . . . . . . . . . . . . . . . . . . . . . . . . . . . 824

2.3.1 The proof of the Adjoint Theorem . . . . . . . . . . . . . . . . . . . . . . . 825

2.3.2 An inverse of the Adjoint Theorem . . . . . . . . . . . . . . . . . . . . . 826

3 Infinitesimal Torelli Theorem for projective hypersurfaces . . . . . . . . . . . . . . . . . . . . 828

3.1 Meromorphic 1-forms on a smooth projective hypersurface . . . . . . . . . . . . . . . . . . 829

3.2 A canonical choice of adjoints on a hypersurface of degree $d>2 \ldots \ldots$. . . . . . . . 831

3.3 The adjoint sublinear systems obtained by meromorphic 1 -forms . . . . . . . . . . . . . . 833

3.4 On Griffiths's proof of infinitesimal Torelli Theorem . . . . . . . . . . . . . . . . . . . . . . . 834

References . . . . . . . . . . . . . . . . . . . . . . . 835

$凶$ Luca Rizzi

rizzi.luca@spes.uniud.it

Francesco Zucconi

francesco.zucconi@uniud.it

1 D.I.M.I., The University of Udine, 33100 Udine, Italy 


\section{Introduction}

Let $X$ be an $m$-dimensional smooth projective variety and $\mathcal{F}$ be a rank $n$ locally free sheaf over it. A way to study $\mathcal{F}$ is to study its extensions $0 \rightarrow \mathcal{L} \rightarrow \mathcal{E} \rightarrow \mathcal{F} \rightarrow 0$ which, up to isomorphism, are parametrized by $\operatorname{Ext}^{1}(\mathcal{F}, \mathcal{L})$. In [2,3,5,6,10-13] and [1] the adjoint forms associated to $\xi \in \operatorname{Ext}^{1}\left(\mathcal{O}_{X}, \mathcal{F}\right)$ are deeply studied and many applications are given. Let us recall the notion of adjoint form in the case $\mathcal{L}=\mathcal{O}_{X}$.

Given $\xi \in \operatorname{Ext}^{1}\left(\mathcal{O}_{X}, \mathcal{F}\right)$, take an $(n+1)$-dimensional subspace $W$ of the kernel of the cup-product homomorphism $\partial_{\xi}: H^{0}(X, \mathcal{F}) \rightarrow H^{1}\left(X, \mathcal{O}_{X}\right)$. Denote by $\lambda^{i} W$ the image of $\bigwedge^{i} W$ through the natural homomorphism $\lambda^{i}: \bigwedge^{i} H^{0}(X, \mathcal{F}) \rightarrow H^{0}\left(X, \bigwedge^{i} \mathcal{F}\right)$. If $\mathcal{B}:=$ $\left\langle\eta_{1}, \ldots, \eta_{n+1}\right\rangle$ is a basis of $W$ and $s_{1}, \ldots, s_{n+1} \in H^{0}(X, \mathcal{E})$ are liftings of $\eta_{1}, \ldots, \eta_{n+1}$, respectively, then the map $\Lambda^{n+1}: \bigwedge^{n+1} H^{0}(X, \mathcal{E}) \rightarrow H^{0}\left(X, \bigwedge^{n+1} \mathcal{E}\right)$ gives the top form $\Omega:=\Lambda^{n+1}\left(s_{1} \wedge s_{2} \wedge \ldots \wedge s_{n+1}\right) \in H^{0}(X, \operatorname{det} \mathcal{E})$. The section $\Omega$ corresponds to a top form $\omega_{\xi, W, \widehat{\mathcal{B}}} \in H^{0}(X, \operatorname{det} \mathcal{F})$ via the isomorphism $\operatorname{det} \mathcal{F} \simeq \operatorname{det} \mathcal{E}$, where $\widehat{\mathcal{B}}=\left\langle s_{1}, \ldots, s_{n+1}\right\rangle$; the form $\omega_{\xi, W, \widehat{\mathcal{B}}}$ is called an adjoint form of $W$ and $\xi$. To the basis $\mathcal{B}$ there are also naturally associated $n+1$ elements $\omega_{i}:=\lambda^{n}\left(\eta_{1} \wedge \ldots \wedge \eta_{i-1} \wedge \widehat{\eta_{i}} \wedge \eta_{i+1} \wedge \ldots \wedge \eta_{n+1}\right), i=$ $1, \ldots, n+1$, obtained by the basis $\left\langle\eta_{1} \wedge \ldots \wedge \eta_{i-1} \wedge \widehat{\eta}_{i} \wedge \eta_{i+1} \wedge \ldots \wedge \eta_{n+1}\right\rangle_{i=1}^{n+1}$ of $\bigwedge^{n} W$. Note that if we change the liftings $s_{1}, \ldots, s_{n+1} \in H^{0}(X, \mathcal{E})$ with other liftings $\widetilde{s}_{1}, \ldots, \widetilde{s}_{n+1}$, then $\omega_{\xi, W, \widehat{\mathcal{B}}}$ is a linear combination of $\omega_{\xi, W, \widetilde{\mathcal{B}}}$ and $\omega_{1}, \ldots, \omega_{n+1}$. The natural problem of this theory is to characterize the condition $\omega_{\xi, W, \widehat{\mathcal{B}}} \in \lambda^{n} W$ in terms of the fixed divisor $D_{W}$ of $\left|\lambda^{n} W\right| \subset \mathbb{P} H^{0}(X$, det $\mathcal{F})$ and of the base locus $Z_{W}$ of the moving part $M_{W} \in \mathbb{P} H^{0}\left(X, \operatorname{det} \mathcal{F} \otimes_{\mathcal{O}_{X}} \mathcal{O}_{X}\left(-D_{W}\right)\right)$, where $\left|\lambda^{n} W\right|=D_{W}+\left|M_{W}\right|$.

In this paper we consider the general case where $\mathcal{L}$ is an invertible sheaf not necessarily equal to $\mathcal{O}_{X}$. In this case $\operatorname{det} \mathcal{E}=\mathcal{L} \otimes \operatorname{det} \mathcal{F}$ and liftings $s_{1}, \ldots, s_{n+1} \in H^{0}(X, \mathcal{E})$ of $\eta_{1}, \ldots, \eta_{n+1} \in H^{0}(X, \mathcal{F})$ determine $\Omega:=\Lambda^{n+1}\left(s_{1} \wedge s_{2} \wedge \ldots \wedge s_{n+1}\right) \in H^{0}(X, \operatorname{det} \mathcal{E})$ which is now called a generalized adjoint form. We define as before $\omega_{i}:=\lambda^{n}\left(\eta_{1} \wedge \ldots \wedge\right.$ $\left.\eta_{i-1} \wedge \widehat{\eta}_{i} \wedge \eta_{i+1} \wedge \ldots \wedge \eta_{n+1}\right), i=1, \ldots, n+1$ and we characterize the case where $\Omega$ belongs to the image of $H^{0}(X, \mathcal{L}) \otimes \lambda^{n} W \rightarrow H^{0}(X, \operatorname{det} \mathcal{E})$ by the natural tensor product map. The game is more complicated than in the above-mentioned papers because the linear system $\left|\lambda^{n} W\right|$ is inside $\mathbb{P} H^{0}(X$, det $\mathcal{F})$ and we have to relate the fixed divisor $D_{W}$ of $\left|\lambda^{n} W\right|$ and the base locus $Z_{W}$ of the moving part $M_{W} \in \mathbb{P} H^{0}\left(X, \operatorname{det} \mathcal{F} \otimes_{\mathcal{O}_{X}} \mathcal{O}_{X}\left(-D_{W}\right)\right)$ to forms which are not anymore inside $H^{0}(X$, det $\mathcal{F})$. Nevertheless the result is analogue to the one of [11, Theorem 1.5.1] and [13, Theorem 2.1.7]:

Theorem [A] Let $X$ be an $m$-dimensional complex compact smooth variety. Let $\mathcal{F}$ be a rank $n$ locally free sheaf on $X$ and $\mathcal{L}$ an invertible sheaf. Consider an extension $0 \rightarrow \mathcal{L} \rightarrow \mathcal{E} \rightarrow$ $\mathcal{F} \rightarrow 0$ corresponding to $\xi \in \operatorname{Ext}^{1}(\mathcal{F}, \mathcal{L})$. Let $W=\left\langle\eta_{1}, \ldots, \eta_{n+1}\right\rangle$ be an $n+1$-dimensional sublinear system of $\operatorname{ker}\left(\partial_{\xi}\right) \subset H^{0}(X, \mathcal{F})$. Let $\Omega \in H^{0}(X, \operatorname{det} \mathcal{E})$ be a generalized adjoint form associated to $W$ as above. It holds that if $\Omega \in \operatorname{Im}\left(H^{0}(X, \mathcal{L}) \otimes \lambda^{n} W \rightarrow H^{0}(X\right.$, det $\left.\mathcal{E})\right)$ then $\xi \in \operatorname{ker}\left(H^{1}\left(X, \mathcal{F}^{\vee} \otimes \mathcal{L}\right) \rightarrow H^{1}\left(X, \mathcal{F}^{\vee} \otimes \mathcal{L}\left(D_{W}\right)\right)\right)$.

Theorem [A], called Adjoint Theorem, can be thought as a general version of the wellknown Castelnuovo's free pencil trick; c.f. see Theorem 2.8.

We have also a viceversa of the Adjoint Theorem; see: Theorem 2.9:

Theorem [B] Under the same hypothesis of Theorem [A], assume also that $H^{0}(X, \mathcal{L}) \cong$ $H^{0}\left(X, \mathcal{L}\left(D_{W}\right)\right)$. It holds that if $\xi \in \operatorname{ker}\left(H^{1}\left(X, \mathcal{F}^{\vee} \otimes \mathcal{L}\right) \rightarrow H^{1}\left(X, \mathcal{F}^{\vee} \otimes \mathcal{L}\left(D_{W}\right)\right)\right)$, then $\Omega \in \operatorname{Im}\left(H^{0}(X, \mathcal{L}) \otimes \lambda^{n} W \rightarrow H^{0}(X, \operatorname{det} \mathcal{E})\right)$.

In particular in the case $D_{W}=0$ Theorem [B] is a full characterization of the condition $\Omega \in \operatorname{Im}\left(H^{0}(X, \mathcal{L}) \otimes \lambda^{n} W \rightarrow H^{0}(X, \operatorname{det} \mathcal{E})\right)$. 
Now by the Adjoint Theorem and by Theorem [B] we can study extension classes of sheaves via adjoint forms. Indeed even if $\mathcal{F}$ has no global sections we can always take the tensor product with a sufficiently ample linear system $\mathcal{M}$ such that $\mathcal{F} \otimes \mathcal{M}$ has enough global sections in order to apply the theory of adjoint forms. By applying the above idea to the case where $n>2, X \subset \mathbb{P}^{n}$ is an hypersurface of degree $d>3$ and $\mathcal{F}:=\Omega_{X}^{1} \otimes_{\mathcal{O}_{X}} \mathcal{O}_{X}(2)$ we have a reformulation of the infinitesimal Torelli Theorem for $X$ in the setting of generalized adjoint theory. In this paper we will not recall the theory concerning infinitesimal Torelli Theorems, for which a reference is [16], in any case a quick introduction to this topic is also given in [13]. Here we point out only that given a degree $d$ form $F \in \mathbb{C}\left[\xi_{0}, \ldots, \xi_{n}\right]$ the Jacobian ideal of $F$ is the ideal $\mathcal{J}$ generated by the partial derivatives $\frac{\partial F}{\partial \xi_{i}}$ for $i=0, \ldots, n$ and by [9][Theorem 9.8], any infinitesimal deformation $\xi \in H^{1}\left(X, \Theta_{X}\right)$, where $X=(F=0)$ and $\Theta_{X}$ is the sheaf of tangent vectors on $X$, is given by a class $[R]$ in the quotient $\mathbb{C}\left[\xi_{0}, \ldots, \xi_{n}\right] / \mathcal{J}$ where $R$ is a homogeneous form of degree $d$.

Theorem [C] For a smooth hypersurface $X$ of degree $d$ in $\mathbb{P}^{n}$ with $n \geq 3$ and $d>3$ the following are equivalent:

(1) the differential of the period map is zero on the infinitesimal deformation

$$
[R] \in\left(\mathbb{C}\left[\xi_{0}, \ldots, \xi_{n}\right] / \mathcal{J}\right)_{d} \simeq H^{1}\left(X, \Theta_{X}\right)
$$

(2) $R$ is an element of the Jacobian ideal $\mathcal{J}$

(3) $\Omega \in \operatorname{Im}\left(H^{0}\left(X, \mathcal{O}_{X}(2)\right) \otimes \lambda^{n} W \rightarrow H^{0}\left(X, \mathcal{O}_{X}(n+d-1)\right)\right)$ for the generic generalized adjoint $\Omega$

(4) The generic generalized adjoint $\Omega$ lies in $\mathcal{J}$.

Note that Theorem $[\mathrm{C}]$ has a different flavor with respect to the analogue [9, Theorem 9.8] since we essentially use meromorphic 1-forms over $X$; see Proposition 3.7. Finally we want to mention that in a forthcoming paper [14] we show how to recover also the Green's infinitesimal Torelli Theorem for a sufficiently ample divisor of a smooth variety in terms of generalized adjoint theory.

\section{The theory of generalized adjoint forms}

\subsection{Definition of generalized adjoint form}

Let $X$ be a smooth compact complex variety of dimension $m$ and let $\mathcal{F}$ and $\mathcal{L}$ be two locally free sheaves on $X$ of rank $n$ and 1, respectively. Consider the exact sequence of locally free sheaves

$$
0 \rightarrow \mathcal{L} \rightarrow \mathcal{E} \rightarrow \mathcal{F} \rightarrow 0
$$

associated to an element $\xi \in \operatorname{Ext}^{1}(\mathcal{F}, \mathcal{L}) \cong H^{1}\left(X, \mathcal{F}^{\vee} \otimes \mathcal{L}\right)$. Recall that the invertible sheaf $\operatorname{det} \mathcal{F}:=\bigwedge^{n} \mathcal{F}$ fits into the exact sequence

$$
0 \rightarrow \bigwedge^{n-1} \mathcal{F} \otimes \mathcal{L} \rightarrow \bigwedge^{n} \mathcal{E} \rightarrow \operatorname{det} \mathcal{F} \rightarrow 0
$$

which still corresponds to $\xi$ under the isomorphism $\operatorname{Ext}^{1}(\mathcal{F}, \mathcal{L}) \cong \operatorname{Ext}^{1}\left(\operatorname{det} \mathcal{F}, \bigwedge^{n-1} \mathcal{F} \otimes\right.$ $\mathcal{L}) \cong H^{1}\left(X, \mathcal{F}^{\vee} \otimes \mathcal{L}\right)$. Furthermore $\operatorname{det} \mathcal{F}$ satisfies

$$
\operatorname{det} \mathcal{F} \otimes \mathcal{L} \cong \operatorname{det} \mathcal{E} .
$$


Let $\partial_{\xi}: H^{0}(X, \mathcal{F}) \rightarrow H^{1}(X, \mathcal{L})$ be the connecting homomorphism related to (2.1), and let $W \subset \operatorname{ker}\left(\partial_{\xi}\right)$ be a vector subspace of dimension $n+1$. Choose a basis $\mathcal{B}:=\left\{\eta_{1}, \ldots, \eta_{n+1}\right\}$ of $W$. By definition we can take liftings $s_{1}, \ldots, s_{n+1} \in H^{0}(X, \mathcal{E})$ of the sections $\eta_{1}, \ldots, \eta_{n+1}$. If we consider the natural map

$$
\Lambda^{n}: \bigwedge^{n} H^{0}(X, \mathcal{E}) \rightarrow H^{0}\left(X, \bigwedge^{n} \mathcal{E}\right)
$$

we can define the sections

$$
\Omega_{i}:=\Lambda^{n}\left(s_{1} \wedge \ldots \wedge \hat{s_{i}} \wedge \ldots \wedge s_{n+1}\right)
$$

for $i=1, \ldots, n+1$. Denote by $\omega_{i}$, for $i=1, \ldots, n+1$, the corresponding sections in $H^{0}(X$, det $\mathcal{F})$. Obviously we have that $\omega_{i}=\lambda^{n}\left(\eta_{1} \wedge \ldots \wedge \hat{\eta}_{i} \wedge \ldots \wedge \eta_{n+1}\right)$, where $\lambda^{n}$ is the natural morphism

$$
\lambda^{n}: \bigwedge^{n} H^{0}(X, \mathcal{F}) \rightarrow H^{0}(X, \operatorname{det} \mathcal{F})
$$

The vector subspace of $H^{0}(X$, det $\mathcal{F})$ generated by $\omega_{1}, \ldots, \omega_{n+1}$ is denoted by $\lambda^{n} W$.

Definition 2.1 If $\lambda^{n} W$ is nontrivial, it induces a sublinear system $\left|\lambda^{n} W\right| \subset \mathbb{P}\left(H^{0}(X\right.$, det $\left.\mathcal{F})\right)$ that we will call adjoint sublinear system. We call $D_{W}$ its fixed divisor and $Z_{W}$ the base locus of its moving part $\left|M_{W}\right| \subset \mathbb{P}\left(H^{0}\left(X, \operatorname{det} \mathcal{F}\left(-D_{W}\right)\right)\right)$.

Definition 2.2 The section $\Omega \in H^{0}(X, \operatorname{det} \mathcal{E})$ corresponding to $s_{1} \wedge \ldots \wedge s_{n+1}$ via

$$
\Lambda^{n+1}: \bigwedge^{n+1} H^{0}(X, \mathcal{E}) \rightarrow H^{0}(X, \operatorname{det} \mathcal{E})
$$

is called generalized adjoint form.

Remark 2.3 It is easy to see by local computation that this section is in the image of the natural injection $\operatorname{det} \mathcal{E}\left(-D_{W}\right) \otimes \mathcal{I}_{Z_{W}} \rightarrow \operatorname{det} \mathcal{E}$.

We want to study the condition

$$
\Omega \in \operatorname{Im}\left(H^{0}(X, \mathcal{L}) \otimes\left\langle\Omega_{i}\right\rangle \rightarrow H^{0}(X, \operatorname{det} \mathcal{E})\right)
$$

or, equivalently,

$$
\Omega \in \operatorname{Im}\left(H^{0}(X, \mathcal{L}) \otimes \lambda^{n} W \rightarrow H^{0}(X, \operatorname{det} \mathcal{E})\right) .
$$

The first map is given by the wedge product, the second one by (2.3). Note that if $H^{0}(X, \mathcal{L})=$ 0 this condition is equivalent to $\Omega=0$.

Remark 2.4 The choice of the liftings is not relevant for this purpose. Take different liftings $s_{1}^{\prime}, \ldots, s_{n+1}^{\prime} \in H^{0}(X, \mathcal{E})$ of $\eta_{1}, \ldots, \eta_{n+1}$ and call $\Omega_{i}^{\prime} \in H^{0}\left(X, \bigwedge^{n} \mathcal{E}\right)$ and $\Omega^{\prime} \in$ $H^{0}(X, \operatorname{det} \mathcal{E})$ the corresponding sections constructed as above. Obviously

$$
\operatorname{Im}\left(H^{0}(X, \mathcal{L}) \otimes\left\langle\Omega_{i}\right\rangle \rightarrow H^{0}(X, \operatorname{det} \mathcal{E})\right)=\operatorname{Im}\left(H^{0}(X, \mathcal{L}) \otimes\left\langle\Omega_{i}^{\prime}\right\rangle \rightarrow H^{0}(X, \operatorname{det} \mathcal{E})\right),
$$

since they are both equal to $\operatorname{Im}\left(H^{0}(X, \mathcal{L}) \otimes \lambda^{n} W \rightarrow H^{0}(X, \operatorname{det} \mathcal{E})\right)$. It is also easy to see that $\Omega \in \operatorname{Im}\left(H^{0}(X, \mathcal{L}) \otimes\left\langle\Omega_{i}\right\rangle \rightarrow H^{0}(X, \operatorname{det} \mathcal{E})\right)$ iff $\Omega^{\prime} \in \operatorname{Im}\left(H^{0}(X, \mathcal{L}) \otimes\left\langle\Omega_{i}^{\prime}\right\rangle \rightarrow\right.$ $\left.H^{0}(X, \operatorname{det} \mathcal{E})\right)$. 
Remark 2.5 Consider another basis $\mathcal{B}^{\prime}:=\left\{\eta_{1}^{\prime}, \ldots, \eta_{n+1}^{\prime}\right\}$ of $W$ and let $A$ be the matrix of the basis change. The sections $s_{1}^{\prime}, \ldots, s_{n+1}^{\prime}$ obtained from $s_{1}, \ldots, s_{n+1}$ through the matrix $A$ are liftings of $\eta_{1}^{\prime}, \ldots, \eta_{n+1}^{\prime}$. The section $\Omega^{\prime}:=\Lambda^{n+1}\left(s_{1}^{\prime} \wedge \ldots \wedge s_{n+1}^{\prime}\right)$ satisfies $\Omega^{\prime}=\operatorname{det} A \cdot \Omega$. Moreover $\Omega \in \operatorname{Im}\left(H^{0}(X, \mathcal{L}) \otimes\left\langle\Omega_{i}\right\rangle \rightarrow H^{0}(X, \operatorname{det} \mathcal{E})\right)$ iff $\Omega^{\prime} \in \operatorname{Im}\left(H^{0}(X, \mathcal{L}) \otimes\left\langle\Omega_{i}^{\prime}\right\rangle \rightarrow\right.$ $\left.H^{0}(X, \operatorname{det} \mathcal{E})\right)$.

Lemma 2.6 If $\Omega \in \operatorname{Im}\left(H^{0}(X, \mathcal{L}) \otimes\left\langle\Omega_{i}\right\rangle \rightarrow H^{0}(X, \operatorname{det} \mathcal{E})\right)$, then we can find liftings $\tilde{s_{i}} \in H^{0}(X, \mathcal{E}), i=1, \ldots, n+1$, such that $\tilde{\Omega}:=\Lambda^{n+1}\left(\tilde{s_{1}} \wedge \ldots \wedge \tilde{s}_{n+1}\right)=0$.

Proof By hypothesis there exist $\sigma_{i} \in H^{0}(X, \mathcal{L})$ such that

$$
\Omega=\sum_{i=1}^{n+1} \sigma_{i} \wedge \Omega_{i}
$$

We can define new liftings for the element $\eta_{i}$ :

$$
\tilde{s_{i}}:=s_{i}+(-1)^{n-i} \sigma_{i} .
$$

Now, since

$$
\tilde{s_{1}} \wedge \ldots \wedge \tilde{s}_{n+1}=s_{1} \wedge \ldots \wedge s_{n+1}-\sum_{i=1}^{n+1} s_{1} \wedge \ldots \wedge \hat{s_{i}} \wedge \ldots \wedge s_{n+1} \wedge \sigma_{i}
$$

we immediately deduce $\tilde{\Omega}=0$.

From the natural map

$$
\mathcal{F}^{\vee} \otimes \mathcal{L} \rightarrow \mathcal{F}^{\vee} \otimes \mathcal{L}\left(D_{W}\right)
$$

we have a homomorphism

$$
H^{1}\left(X, \mathcal{F}^{\vee} \otimes \mathcal{L}\right) \stackrel{\rho}{\rightarrow} H^{1}\left(X, \mathcal{F}^{\vee} \otimes \mathcal{L}\left(D_{W}\right)\right) ;
$$

we call $\xi_{D_{W}}=\rho(\xi)$.

\subsection{Castelnuovo's free pencil trick}

Consider the case where both $\mathcal{L}$ and $\mathcal{F}$ are of rank one, while $X$ has dimension $m$. In this case $W=\left\langle\eta_{1}, \eta_{2}\right\rangle \subset H^{0}(X, \mathcal{F})$ has dimension two; as usual we choose liftings $s_{1}, s_{2} \in H^{0}(X, \mathcal{E})$ of $\eta_{1}, \eta_{2}$. Note also that $\omega_{1}=\eta_{2}$ and $\omega_{2}=\eta_{1}$, in particular $W=\lambda^{1} W$ so $D_{W}$ is the fixed part of $W$ and $Z_{W}$ is the base locus of its moving part. Call $\tilde{\eta}_{i} \in H^{0}\left(X, \mathcal{F}\left(-D_{W}\right)\right)$ the sections corresponding to the $\eta_{i}$ 's via $H^{0}\left(X, \mathcal{F}\left(-D_{W}\right)\right) \rightarrow H^{0}(X, \mathcal{F})$. The following lemma is well known and it is the core of the Castelnuovo base point free pencil trick.

Lemma 2.7 We have an exact sequence

$$
0 \rightarrow \mathcal{F}^{\vee}\left(D_{W}\right) \stackrel{i}{\rightarrow} \mathcal{O}_{X} \oplus \mathcal{O}_{X} \stackrel{v}{\rightarrow} \mathcal{F}\left(-D_{W}\right) \otimes \mathcal{I}_{Z_{W}} \rightarrow 0
$$

where the morphism $i$ is given by contraction with $-\tilde{\eta}_{1}$ and $\tilde{\eta}_{2}$, while $v$ is given by evaluation with $\tilde{\eta}_{2}$ on the first component and $\tilde{\eta}_{1}$ on the second one.

It is easy to see by local computation that sequence (2.11) fits into the following commutative diagram 


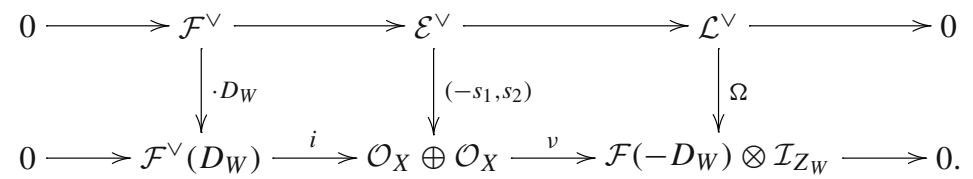

The morphism $\mathcal{E}^{\vee} \rightarrow \mathcal{O}_{X} \oplus \mathcal{O}_{X}$ is given by contraction with the sections $-s_{1}$ and $s_{2}$, the morphism $\mathcal{L}^{\vee} \rightarrow \mathcal{F}\left(-D_{W}\right) \otimes \mathcal{I}_{Z_{W}}$ by contraction with the adjoint $\Omega$. We can prove now the following

Theorem 2.8 Let $X$ be an m-dimensional complex compact smooth variety. Let $\mathcal{F}, \mathcal{L}$ be invertible sheaves on $X$. Consider $\xi \in H^{1}\left(X, \mathcal{F}^{\vee} \otimes \mathcal{L}\right)$ associated to the extension (2.1). Define $W=\left\langle\eta_{1}, \eta_{2}\right\rangle \subset \operatorname{ker}\left(\partial_{\xi}\right) \subset H^{0}(X, \mathcal{F})$ and $\Omega$ as above. We have that $\Omega \in \operatorname{Im}\left(H^{0}(X, \mathcal{L}) \otimes W \rightarrow H^{0}(X, \operatorname{det} \mathcal{E})\right)$ if and only if $\xi_{D_{W}}=0$.

Proof Tensoring (2.12) by $\mathcal{L}$ and passing to cohomology we have the following diagram

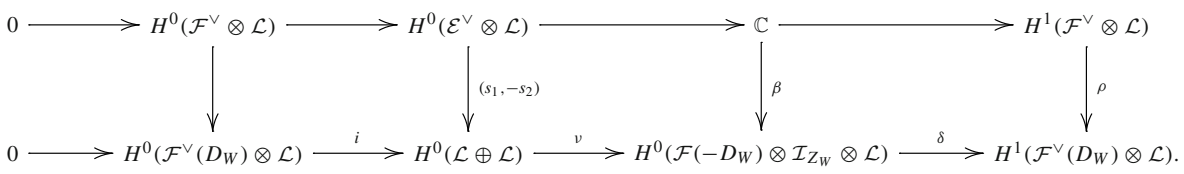

Obviously $\beta(1)=\Omega$ and, by commutativity, $\delta(\beta(1))=\xi_{D_{W}}$. We have then $\xi_{D_{W}}=0$ if and only if $\Omega \in \operatorname{Im}\left(H^{0}(\mathcal{L} \oplus \mathcal{L}) \stackrel{\nu}{\rightarrow} H^{0}\left(\mathcal{F}\left(-D_{W}\right) \otimes \mathcal{I}_{Z_{W}} \otimes \mathcal{L}\right)\right)$. Since $v$ is given by the sections $\tilde{\eta}_{2}$ and $\tilde{\eta}_{1}$, this condition is equivalent to $\Omega \in \operatorname{Im}\left(H^{0}(X, \mathcal{L}) \otimes W \rightarrow H^{0}(X\right.$, det $\left.\mathcal{E})\right)$, since $\operatorname{det} \mathcal{E}=\mathcal{F} \otimes \mathcal{L}$.

\subsection{The Adjoint Theorem}

We go back now to the general case with $\mathcal{F}$ locally free of rank $n$. By obvious identifications the natural map

$$
\operatorname{Ext}^{1}\left(\operatorname{det} \mathcal{F}, \bigwedge^{n-1} \mathcal{F} \otimes \mathcal{L}\right) \rightarrow \operatorname{Ext}^{1}\left(\operatorname{det} \mathcal{F}\left(-D_{W}\right), \bigwedge^{n-1} \mathcal{F} \otimes \mathcal{L}\right)
$$

gives an extension $\mathcal{E}^{(n)}$ and a commutative diagram:

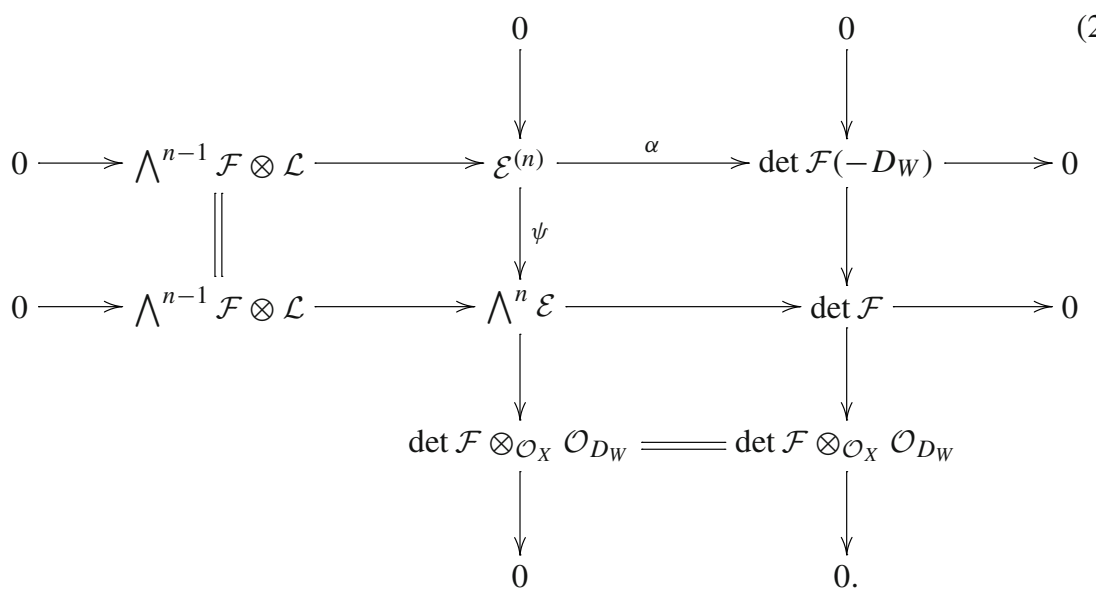




\subsubsection{The proof of the Adjoint Theorem}

By the hypothesis $\Omega \in \operatorname{Im}\left(H^{0}(X, \mathcal{L}) \otimes \lambda^{n} W \rightarrow H^{0}(X, \operatorname{det} \mathcal{E})\right)$ and by lemma (2.6), we can choose liftings $s_{i} \in H^{0}(X, \mathcal{E})$ of $\eta_{i}$ with $\Omega=0$.

Since $D_{W}$ is the fixed divisor of the linear system $\left|\lambda^{n} W\right|$ and the sections $\omega_{i}$ generate this linear system, then the $\omega_{i}$ are in the image of

$$
\operatorname{det} \mathcal{F}\left(-D_{W}\right) \rightarrow \operatorname{det} \mathcal{F}
$$

so we can find sections $\tilde{\omega}_{i} \in H^{0}\left(X, \operatorname{det} \mathcal{F}\left(-D_{W}\right)\right)$ such that

$$
\tilde{\omega}_{i} \cdot d=\omega_{i}
$$

where $d$ is a global section of $\mathcal{O}_{X}\left(D_{W}\right)$ with $(d)=D_{W}$. Hence, using the commutativity of (2.14), we can find liftings $\tilde{\Omega}_{i} \in H^{0}\left(X, \mathcal{E}^{(n)}\right)$ of the sections $\Omega_{i}$. The evaluation map

$$
\bigoplus_{i=1}^{n+1} \mathcal{O}_{X} \stackrel{\tilde{\mu}}{\rightarrow} \mathcal{E}^{(n)}
$$

given by the global sections $\tilde{\Omega}_{i}$, composed with the map $\alpha$ of (2.14), induces a map $\mu$ which fits into the following diagram

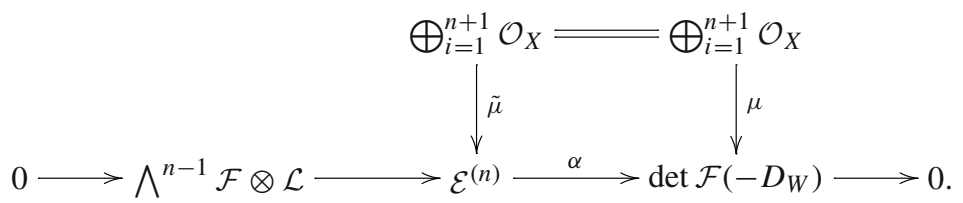

We point out that the morphism $\mu$ is given by multiplication by $\tilde{\omega}_{i}$ on the $i$-th component. The sheaf $\operatorname{Im} \tilde{\mu}$ is torsion free since it is a subsheaf of the locally free sheaf $\mathcal{E}^{(n)}$. Moreover, since $\Omega=0$, a local computation shows that $\operatorname{Im} \tilde{\mu}$ has rank one outside $Z_{W}$. On the other hand the sheaf $\operatorname{Im} \mu$ is by definition

$$
\operatorname{Im} \mu=\operatorname{det} \mathcal{F}\left(-D_{W}\right) \otimes \mathcal{I}_{Z_{W}} .
$$

The morphism

$$
\alpha: \mathcal{E}^{(n)} \rightarrow \operatorname{det} \mathcal{F}\left(-D_{W}\right)
$$

induces a surjective morphism, that we continue to call $\alpha$,

$$
\operatorname{Im} \tilde{\mu} \stackrel{\alpha}{\rightarrow} \operatorname{Im} \mu
$$

between two sheaves that are locally free of rank one outside $Z_{W}$. This morphism is also injective, because its kernel is a torsion subsheaf of the torsion free sheaf $\operatorname{Im} \tilde{\mu}$, hence it is trivial.

We have proved that

$$
\operatorname{Im} \tilde{\mu} \cong \operatorname{det} \mathcal{F}\left(-D_{W}\right) \otimes \mathcal{I}_{Z_{W}},
$$

so

$$
\mathcal{E}^{(n)} \supset(\operatorname{Im} \tilde{\mu})^{\vee \vee} \cong \operatorname{det} \mathcal{F}\left(-D_{W}\right) .
$$

This isomorphism gives the splitting

$$
0 \longrightarrow \wedge^{n-1} \mathcal{F} \otimes \mathcal{L} \longrightarrow \mathcal{E}^{(n)} \longrightarrow \operatorname{det} \mathcal{F}\left(-D_{W}\right) \longrightarrow 0 .
$$


Since $\xi_{D_{W}}$ is the element of $H^{1}\left(X, \mathcal{F}^{\vee} \otimes \mathcal{L}\left(D_{W}\right)\right)$ associated to this extension, we conclude that $\xi_{D_{W}}=0$.

We have proved the Adjoint Theorem.

\subsubsection{An inverse of the Adjoint Theorem}

We prove now an inverse of the Adjoint Theorem.

Theorem 2.9 Let $X$ be an m-dimensional complex compact smooth variety. Let $\mathcal{F}$ be a rank $n$ locally free sheaf on $X$ and $\mathcal{L}$ an invertible sheaf. Consider an extension $0 \rightarrow$ $\mathcal{L} \rightarrow \mathcal{E} \rightarrow \mathcal{F} \rightarrow 0$ corresponding to $\xi \in \operatorname{Ext}^{1}(\mathcal{F}, \mathcal{L})$. Let $W=\left\langle\eta_{1}, \ldots, \eta_{n+1}\right\rangle$ be a $n+1$-dimensional sublinear system of $\operatorname{ker}\left(\partial_{\xi}\right) \subset H^{0}(X, \mathcal{F})$. Let $\Omega \in H^{0}(X, \operatorname{det} \mathcal{E})$ be an adjoint form associated to $W$ as above. Assume that $H^{0}(X, \mathcal{L}) \cong H^{0}\left(X, \mathcal{L}\left(D_{W}\right)\right)$. If $\xi \in \operatorname{ker}\left(H^{1}\left(X, \mathcal{F}^{\vee} \otimes \mathcal{L}\right) \rightarrow H^{1}\left(X, \mathcal{F}^{\vee} \otimes \mathcal{L}\left(D_{W}\right)\right)\right)$, then $\Omega \in \operatorname{Im}\left(H^{0}(X, \mathcal{L}) \otimes \lambda^{n} W \rightarrow\right.$ $\left.H^{0}(X, \operatorname{det} \mathcal{E})\right)$.

Proof If $\mathcal{F}$ is a rank one sheaf, then (2.8) gives the thesis without the extra assumption $H^{0}(X, \mathcal{L}) \cong H^{0}\left(X, \mathcal{L}\left(D_{W}\right)\right)$. We assume then rank $\mathcal{F} \geq 2$.

By (2.3), we can write $(\Omega)=D_{W}+F$ with $F$ effective. In the first step of the proof we want to find a global section

$$
\Omega^{\prime} \in H^{0}\left(X, \bigwedge^{n} \mathcal{E} \otimes \mathcal{L}(-F)\right)
$$

which restricts, through the natural map

$$
\bigwedge^{n} \mathcal{E} \otimes \mathcal{L}(-F) \rightarrow \operatorname{det} \mathcal{E}(-F)
$$

to the section $d \in H^{0}(\operatorname{det} \mathcal{E}(-F))$, where $(d)=D_{W}$.

Consider the commutative diagram:

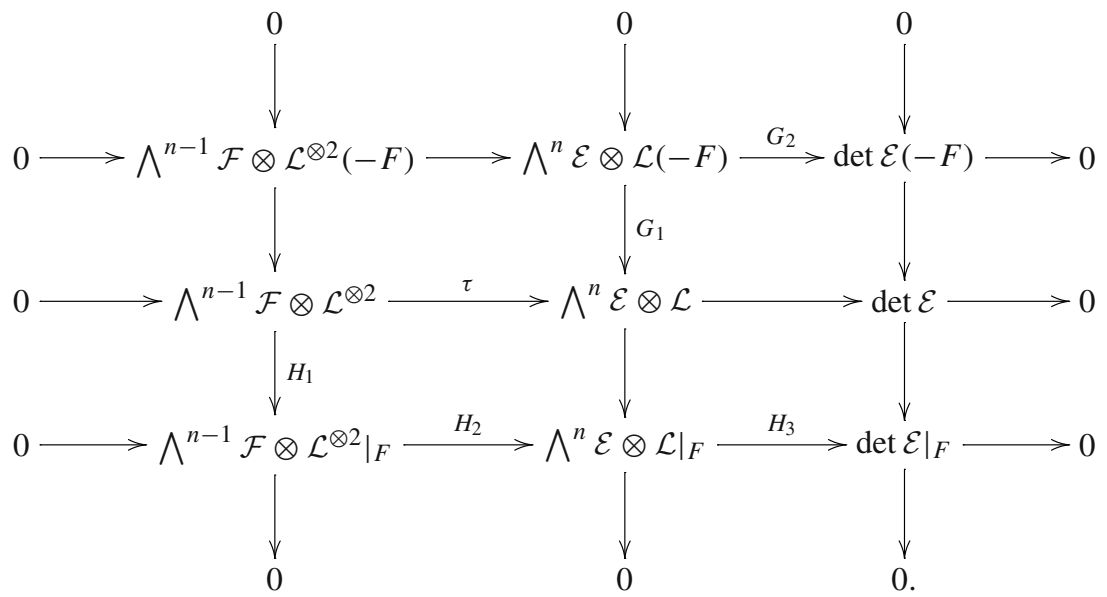

By the hypothesis $\xi_{D_{W}}=0$ it follows easily that there exists a lifting $\tilde{\Omega} \in H^{0}\left(X, \bigwedge^{n} \mathcal{E} \otimes \mathcal{L}\right)$ of $\Omega$. Indeed, tensor (2.14) by $\mathcal{L}$ and take a global lifting $f \in H^{0}\left(X\right.$, $\left.\operatorname{det} \mathcal{E}\left(-D_{W}\right)\right)$ of $\Omega$. Since $\xi_{D_{W}}=0, f$ can be lifted to a section $e \in H^{0}\left(X, \mathcal{E}^{(n)} \otimes \mathcal{L}\right)$. Define $\tilde{\Omega}:=\psi(e)$. By 
commutativity, $H_{3}\left(\left.\tilde{\Omega}\right|_{F}\right)=0$ hence we call $\bar{\mu} \in H^{0}\left(X,\left.\bigwedge^{n-1} \mathcal{F} \otimes \mathcal{L}^{\otimes 2}\right|_{F}\right)$ the lifting of $\left.\tilde{\Omega}\right|_{F}$. A local computation shows that the connecting homomorphism

$$
\delta: H^{0}\left(X,\left.\bigwedge^{n-1} \mathcal{F} \otimes \mathcal{L}^{\otimes 2}\right|_{F}\right) \rightarrow H^{1}\left(X, \bigwedge^{n-1} \mathcal{F} \otimes \mathcal{L}^{\otimes 2}(-F)\right)
$$

maps $\bar{\mu}$ to $\xi_{D_{W}}$, which is zero by hypothesis. Then there exists a global section

$$
\mu \in H^{0}\left(X, \bigwedge^{n-1} \mathcal{F} \otimes \mathcal{L}^{\otimes 2}\right)
$$

which is a lifting of $\bar{\mu}$. The section

$$
\hat{\Omega}:=\Omega-\tau(\mu) \in H^{0}\left(X, \bigwedge^{n} \mathcal{E} \otimes \mathcal{L}\right)
$$

is a new lifting of $\Omega$ that, by construction, vanishes when restricted to $F$. We call

$$
\Omega^{\prime} \in H^{0}\left(X, \bigwedge^{n} \mathcal{E} \otimes \mathcal{L}(-F)\right)
$$

the global section which lifts $\hat{\Omega}$. It is easy to see that $G_{2}\left(\Omega^{\prime}\right)=d$ so $\Omega^{\prime}$ is the section we wanted.

In the second part of the proof we prove that $\Omega \in \operatorname{Im}\left(H^{0}(X, \mathcal{L}) \otimes \lambda^{n} W \rightarrow H^{0}(X, \operatorname{det} \mathcal{E})\right)$. The global sections

$$
\omega_{i}:=\lambda^{n}\left(\eta_{1} \wedge \ldots \wedge \hat{\eta}_{i} \wedge \ldots \wedge \eta_{n+1}\right) \in H^{0}(X, \operatorname{det} \mathcal{F})
$$

generate $\lambda^{n} W$ and by definition they vanish on $D_{W}$, that is there exist global sections $\tilde{\omega}_{i} \in$ $H^{0}\left(X, \operatorname{det} \mathcal{F}\left(-D_{W}\right)\right)$ such that

$$
\omega_{i}=\tilde{\omega}_{i} \cdot d
$$

We consider the commutative diagram

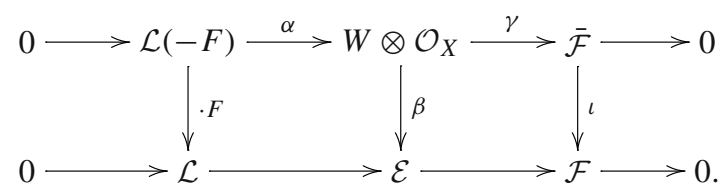

The map $\beta$ is locally defined by

$$
\left(f_{1}, \ldots, f_{n+1}\right) \mapsto(-1)^{n} f_{1} \cdot s_{1}+\cdots+f_{n+1} \cdot s_{n+1} .
$$

The map $\alpha$ is defined in the following way: if $f \in \mathcal{L}(-F)(U)$ is a local section, then, locally on $U, \alpha$ is given by

$$
f \mapsto\left(\tilde{\omega}_{1}(f), \ldots, \tilde{\omega}_{n+1}(f)\right),
$$

where we observe that the sections $\tilde{\omega}_{i}$ are global sections of the dual sheaf of $\mathcal{L}(-F)$. The sheaf $\overline{\mathcal{F}}$ is by definition the cokernel of the first row. Now, tensoring by $\mathcal{L}^{\vee}$, we have

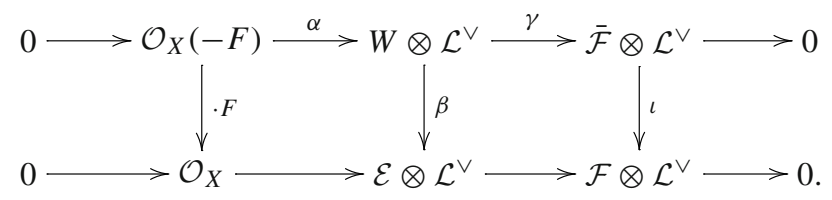

Dualizing and tensoring again by $\mathcal{O}_{X}\left(D_{W}\right)$, we obtain the commutative square 


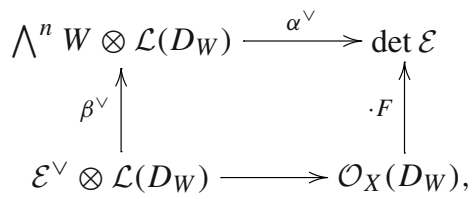

where we have used the isomorphism of vector spaces $W^{\vee} \cong \bigwedge^{n} W$, given by

$$
\eta^{i} \mapsto \eta_{1} \wedge \ldots \wedge \hat{\eta}_{i} \wedge \ldots \wedge \eta_{n+1}=: e_{i}
$$

where $\eta^{1}, \ldots, \eta^{n+1}$ is the basis of $W^{\vee}$ dual to the basis $\eta_{1}, \ldots, \eta_{n+1}$ of $W$. By definition of $\alpha$ we have that $\alpha^{\vee}$ is the evaluation map given by the global sections $\tilde{\omega}_{i}$. Note that $\mathcal{E}^{\vee} \otimes \mathcal{L}\left(D_{W}\right) \cong \bigwedge^{n} \mathcal{E} \otimes \mathcal{L}(-F)$. Taking global sections we have

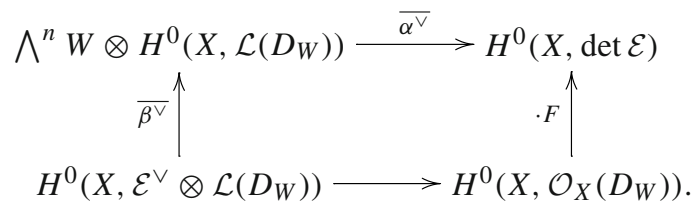

The section $\Omega^{\prime} \in H^{0}\left(X, \mathcal{E}^{\vee} \otimes \mathcal{L}\left(D_{W}\right)\right)$ produces in $H^{0}(X, \operatorname{det} \mathcal{E})$ the adjoint $\Omega$, so by commutativity

$$
\Omega=\overline{\alpha^{\vee}}\left(\overline{\beta^{\vee}}\left(\Omega^{\prime}\right)\right) .
$$

We have

$$
\overline{\beta^{\vee}}\left(\Omega^{\prime}\right)=\sum_{i=1}^{n+1} c_{i} \cdot e_{i} \otimes \sigma_{i},
$$

where $c_{i} \in \mathbb{C}$ and $\sigma_{i} \in H^{0}\left(X, \mathcal{L}\left(D_{W}\right)\right)$. By our hypothesis $H^{0}(X, \mathcal{L}) \cong H^{0}\left(X, \mathcal{L}\left(D_{W}\right)\right)$, there exists sections $\tilde{\sigma}_{i} \in H^{0}(X, \mathcal{L})$ with $\sigma_{i}=\tilde{\sigma}_{i} \cdot d$. So

$\Omega=\overline{\alpha^{\vee}}\left(\overline{\beta^{\vee}}\left(\Omega^{\prime}\right)\right)=\overline{\alpha^{\vee}}\left(\sum_{i=1}^{n+1} c_{i} \cdot e_{i} \otimes \sigma_{i}\right)=\sum_{i=1}^{n+1} c_{i} \cdot \tilde{\omega}_{i} \cdot \sigma_{i}=\sum_{i=1}^{n+1} c_{i} \cdot \tilde{\omega}_{i} \cdot d \cdot \tilde{\sigma}_{i}=\sum_{i=1}^{n+1} c_{i} \cdot \omega_{i} \cdot \tilde{\sigma}_{i}$.

This is exactly our thesis.

By the Adjoint Theorem and (2.9) we deduce the following

Corollary 2.10 If $D_{W}=0$, then $\xi=0$ iff $\Omega \in \operatorname{Im}\left(H^{0}(X, \mathcal{L}) \otimes \lambda^{n} W \rightarrow H^{0}(X, \operatorname{det} \mathcal{E})\right)$.

\section{Infinitesimal Torelli Theorem for projective hypersurfaces}

In this section we want to study adjoint images in the case of smooth hypersurfaces of the projective space $\mathbb{P}^{n}$. 


\subsection{Meromorphic 1-forms on a smooth projective hypersurface}

Let $V \subset \mathbb{P}^{n}$ be a smooth hypersurface defined by a homogeneous polynomial $F \in$ $\mathbb{C}\left[\xi_{0}, \ldots, \xi_{n}\right]$ of degree $\operatorname{deg} F=d$. An infinitesimal deformation $\xi \in \operatorname{Ext}^{1}\left(\Omega_{V}^{1}, \mathcal{O}_{V}\right)$ of $V$ gives an exact sequence for the sheaf of differential forms $\Omega_{V}^{1}$ :

$$
0 \rightarrow \mathcal{O}_{V} \rightarrow \Omega_{\mathcal{V} \mid V}^{1} \rightarrow \Omega_{V}^{1} \rightarrow 0
$$

We assume that $n \geq 3$, hence $H^{0}\left(V, \Omega_{V}^{1}\right)=0$ and we can not construct the adjoint of this sequence directly, so we twist (3.1) by a suitable integer $a$ such that $\Omega_{V}^{1}(a)$ has at least $n=\operatorname{rank}\left(\Omega_{V}^{1}\right)+1$ global sections. A standard computation shows that $a=2$ is enough for this purpose, so from now on we will consider the sequence

$$
0 \rightarrow \mathcal{O}_{V}(2) \rightarrow \Omega_{\mathcal{V} \mid V}^{1}(2) \rightarrow \Omega_{V}^{1}(2) \rightarrow 0
$$

which again corresponds to $\xi \in \operatorname{Ext}^{1}\left(\Omega_{V}^{1}(2), \mathcal{O}_{V}(2)\right) \cong \operatorname{Ext}^{1}\left(\Omega_{V}^{1}, \mathcal{O}_{V}\right) \cong H^{1}\left(V, \Theta_{V}\right)$, where $\Theta_{V}$ denotes the sheaf of vector fields on $V$. Denote by $\mathcal{J}$ the Jacobian ideal of $F$, that is the ideal of $\mathbb{C}\left[\xi_{0}, \ldots, \xi_{n}\right]$ generated by the partial derivatives $\frac{\partial F}{\partial \xi_{i}}$ for $i=0, \ldots, n$. Following [9][Theorem 9.8], the deformation $\xi$ is given by a class [R] of degree $d$ in the quotient $\mathbb{C}\left[\xi_{0}, \ldots, \xi_{n}\right] / \mathcal{J}$. If we choose a representative $R$ of degree $d$ for this class, then $F+t R=0$, for small $t$, is the equation of the hypersurface that is the associated deformation of $V$.

Together with (3.2), we have the conormal exact sequence

$$
0 \rightarrow \mathcal{O}_{V}(-d) \rightarrow \Omega_{\mathbb{P}^{n} \mid V}^{1} \rightarrow \Omega_{V}^{1} \rightarrow 0
$$

If we put these sequences together we obtain the diagram

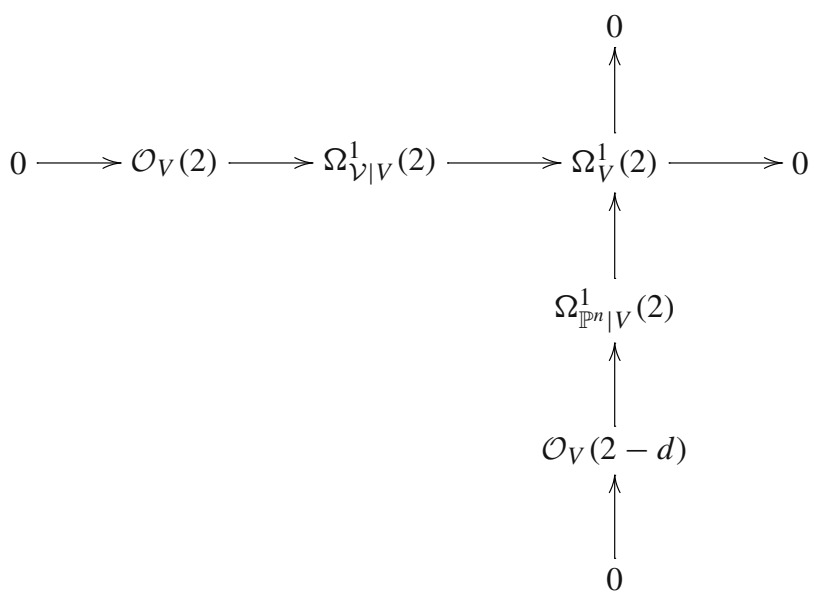


which can be completed as follows

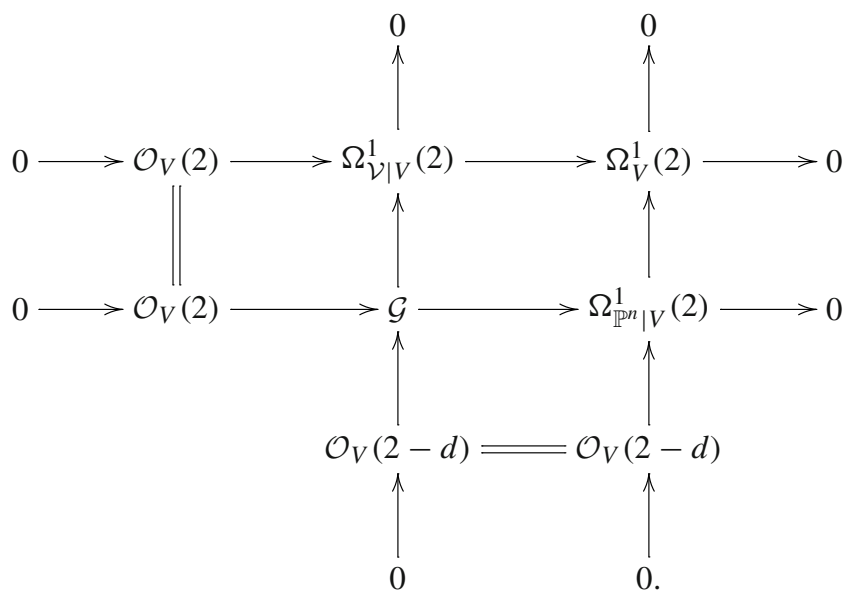

By [9] the deformation $\xi$ of (3.2) comes from $R \in H^{0}\left(\mathbb{P}^{n}, \mathcal{O}_{\mathbb{P}}^{n}(d)\right)$, then it gives the zero element in $H^{0}\left(V, \Theta_{\mathbb{P}^{n} \mid V}\right)$, hence we have that the sheaf $\mathcal{G}$ in (3.4) is a direct sum $\mathcal{G}=\mathcal{O}_{V}(2) \oplus \Omega_{\mathbb{P}^{n} \mid V}^{1}(2)$ and we have a natural morphism $\phi: \Omega_{\mathbb{P}^{n} \mid V}^{1}(2) \rightarrow \Omega_{\mathcal{V} \mid V}^{1}(2)$ which fits in the diagram

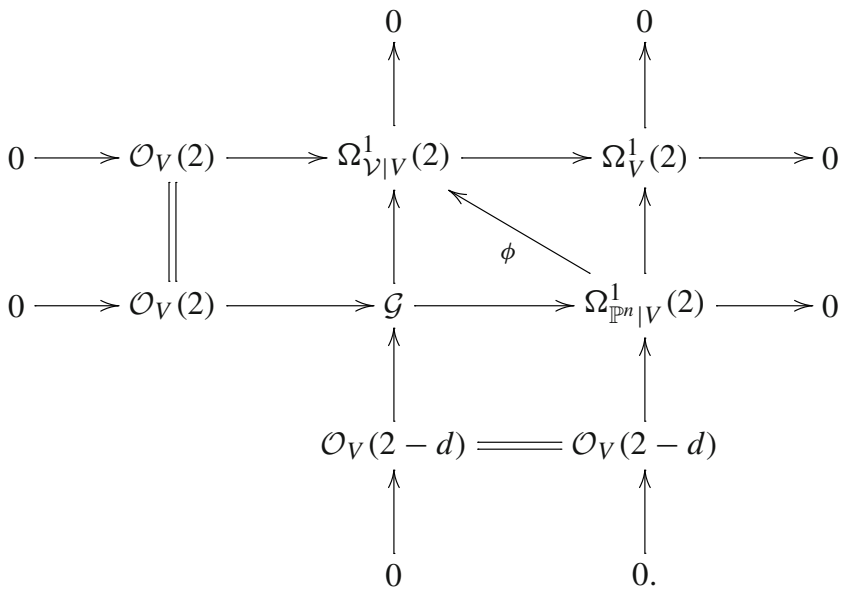

The morphism $\phi$ gives in a natural way a morphism

$$
\begin{aligned}
\phi^{n} & : H^{0}\left(V, \operatorname{det}\left(\Omega_{\mathbb{P} \mid V}^{1}(2)\right)\right) \cong H^{0}\left(V, \mathcal{O}_{V}(n-1)\right) \rightarrow H^{0}\left(V, \operatorname{det}\left(\Omega_{\mathcal{V} \mid V}^{1}(2)\right)\right) \\
& \cong H^{0}\left(V, \mathcal{O}_{V}(n+d-1)\right) .
\end{aligned}
$$

We can write explicitly the isomorphism between $H^{0}\left(V, \operatorname{det}\left(\Omega_{\mathbb{P}^{n} \mid V}^{1}(2)\right)\right)=H^{0}\left(V, \Omega_{\mathbb{P}^{n} \mid V}^{n}\right.$ $(2 n))$ and $H^{0}\left(V, \mathcal{O}_{V}(n-1)\right)$. Note that $H^{0}\left(\mathbb{P}^{n}, \Omega_{\mathbb{P} n}^{n}(2 n)\right) \rightarrow H^{0}\left(V, \Omega_{\mathbb{P} n}^{n}(2 n)\right)$ is surjective, so we will focus on the rational $n$-forms on $\mathbb{P}^{n}$. By [9][Corollary 2.11] this forms may be written as $\omega=\frac{P \Psi}{Q}$ where $\Psi=\sum_{i=0}^{n}(-1)^{i} \xi_{i}\left(d \xi_{0} \wedge \ldots \wedge d \widehat{\xi}_{i} \wedge \ldots \wedge d \xi_{n}\right)$ gives a generator of $H^{0}\left(\mathbb{P}^{n}, \Omega_{\mathbb{p} n}^{n}(n+1)\right)$ and $\operatorname{deg} Q=\operatorname{deg} P+(n+1)$. In our case $Q$ is a polynomial of degree $2 n$, hence $P$ has degree $n-1$. This identification depends on the (noncanonical) choice of the 
polynomial $Q$ and gives an isomorphism $H^{0}\left(V, \Omega_{\mathbb{P}^{n} \mid V}^{n}(2 n)\right) \rightarrow H^{0}\left(V, \mathcal{O}_{V}(n-1)\right)$ defined by $\left.\omega\right|_{V} \mapsto P$.

Proposition 3.1 $\phi^{n}$ is given via the multiplication by the polynomial $R$ (modulo $F$ ).

Proof Locally we can see $\mathcal{V}$ in the product $\Delta \times \mathbb{P}^{n}$ of the projective space with a disk; here $\mathcal{V}$ is defined by the equation $F+t R=0$. Hence $d(F+t R)=0$ in $\Omega_{\mathcal{V}}^{1}$, that is $d F+d t \cdot R+d R \cdot t=0$.

Call $F_{i}:=\frac{\partial F}{\partial \xi_{i}}$. Since $V$ is smooth, there exist $i$ such that $U_{i}=\left(F_{i} \neq 0\right)$ is a nontrivial open subset; let for example $U_{1}$ be nontrivial. Take local coordinates $z_{i}=\frac{\xi_{i}}{\xi_{0}}$ in the open set $\left(\xi_{0} \neq 0\right) \cap U_{1}$. Then we have

$$
d z_{1}=-\frac{R d t}{F_{1}}-\frac{t d R}{F_{1}}-\sum_{i>1} \frac{F_{i}}{F_{1}} d z_{i}
$$

which gives in $V$ (that is for $t=0$ )

$$
d z_{1}=-\frac{R d t}{F_{1}}-\sum_{i>1} \frac{F_{i}}{F_{1}} d z_{i}
$$

The image $\phi^{n}\left(\left.\omega\right|_{V}\right)$ is then obtained by the substitution of (3.7) in $\frac{P(z)}{Q(z)} d z_{1} \wedge \ldots \wedge d z_{n}$, which is the local form of $\frac{P(\xi) \Psi}{Q(\xi)}$. Hence

$$
\frac{P(z)}{Q(z)} d z_{1} \wedge \ldots \wedge d z_{n}=-\frac{P(z) R(z)}{Q(z) F_{1}(z)} d t \wedge d z_{2} \wedge \ldots \wedge d z_{n} .
$$

If we homogenize we obtain on $U_{1}$

$$
\frac{P \Psi}{Q}=-\frac{P R}{Q F_{1}} \sum_{i \neq 1}(-1)^{i-1} \operatorname{sgn}(i-1) \xi_{i} d t \wedge d \xi_{0} \wedge \widehat{d \xi_{1}} \ldots \wedge{\widehat{d \xi_{i}}} \wedge \ldots \wedge d \xi_{n}
$$

Hence

$$
\phi^{n}\left(\left.\omega\right|_{V}\right)=-\frac{P R}{Q F_{1}} \sum_{i \neq 1}(-1)^{i-1} \operatorname{sgn}(i-1) \xi_{i} d t \wedge d \xi_{0} \wedge \widehat{d \xi_{1}} \ldots \wedge{\widehat{d \xi_{i}}} \wedge \ldots \wedge d \xi_{n}
$$

and it is clear that $\phi^{n}$ is given by multiplication with $R$.

\subsection{A canonical choice of adjoints on a hypersurface of degree $d>2$}

We want now to construct adjoint forms associated to the sequence (3.2).

Assume that $n \geq 3$, so that $H^{1}\left(V, \mathcal{O}_{V}(2)\right)=H^{1}\left(V, \mathcal{O}_{V}(2-d)\right)=0$, and we can lift all the global sections of $H^{0}\left(V, \Omega_{V}^{1}(2)\right)$ both in the horizontal and in the vertical sequence of (3.5).

We take $\eta_{1}, \ldots, \eta_{n} \in H^{0}\left(V, \Omega_{V}^{1}(2)\right)$ global forms and we want to find liftings $s_{1}, \ldots, s_{n} \in H^{0}\left(V, \Omega_{\mathcal{V} \mid V}^{1}\right)$. This can be done since $H^{1}\left(V, \mathcal{O}_{V}(2)\right)$ is zero. A generalized adjoint is then the global section of the sheaf $\operatorname{det}\left(\Omega_{\mathcal{V} \mid V}^{1}(2)\right)=\mathcal{O}_{V}(n+d-1)$ given by $\Omega:=\Lambda^{n}\left(s_{1} \wedge \ldots \wedge s_{n}\right) \in H^{0}\left(V, \operatorname{det}\left(\Omega_{\mathcal{V} \mid V}^{1}(2)\right)\right)$.

We point out another interesting way to compute this generalized adjoint form using Proposition (3.1).

Consider the sequence (3.3), that is the vertical sequence in (3.5). Since $H^{1}\left(V, \mathcal{O}_{V}\right.$ $(2-d))=0$, we can find liftings $\tilde{s_{1}}, \ldots, \tilde{s_{n}} \in H^{0}\left(V, \Omega_{\mathbb{P}^{n} \mid V}^{1}(2)\right)$ of the sections $\eta_{1}, \ldots, \eta_{n}$. 
Furthermore they are unique if $d>2$. We can thus consider the adjoint form associated to (3.3) given by $\widetilde{\Omega}:=\Lambda^{n}\left(\tilde{s_{1}} \wedge \ldots \wedge \tilde{s_{n}}\right)$. This adjoint is independent from the deformation $\xi$; it depends only on $V$ and its embedding in $\mathbb{P}^{n}$. If $d>2$, then $\widetilde{\Omega}$ is unique.

To describe $\widetilde{\Omega}$ explicitly we first consider the exact sequence

$$
0 \rightarrow \Omega_{\mathbb{P}^{n}}^{1}(2-d) \rightarrow \Omega_{\mathbb{P}^{n}}^{1}(2) \rightarrow \Omega_{\mathbb{P}^{n} \mid V}^{1}(2) \rightarrow 0 .
$$

If $d>2$, the vanishing of $H^{0}\left(\mathbb{P}^{n}, \Omega_{\mathbb{P}^{n}}^{1}(2-d)\right)$ and $H^{1}\left(\mathbb{P}^{n}, \Omega_{\mathbb{P}^{n}}^{1}(2-d)\right)$ (c.f. Bott Formulas) gives the isomorphism $H^{0}\left(\mathbb{P}^{n}, \Omega_{\mathbb{P}^{n}}^{1}(2)\right)=H^{0}\left(V, \Omega_{\mathbb{P} n \mid V}^{1}(2)\right)$. Hence, the forms $\tilde{s_{i}}$ are the restriction on $V$ of global rational 1-forms. By [9][Theorem 2.9] we can write

$$
\tilde{s_{i}}=\frac{1}{Q} \sum_{j=0}^{n} L_{j}^{i} d \xi_{j}
$$

where $\operatorname{deg} Q=2$ and $L_{j}^{i}$ is a homogeneous polynomial of degree 1 which does not contain $\xi_{j}$ in its expression. Hence

$$
\widetilde{\Omega}=\Lambda^{n}\left(\tilde{s_{1}} \wedge \ldots \wedge \tilde{s_{n}}\right)=\frac{1}{Q^{n}} \sum_{i=0}^{n} M_{i} d \xi_{0} \wedge \ldots \wedge \widehat{d \xi_{i}} \wedge \ldots \wedge d \xi_{n}
$$

where $M_{i}$ is the determinant of the matrix obtained by

$$
\left(\begin{array}{ccc}
L_{0}^{1} & \ldots & L_{0}^{n} \\
\vdots & & \vdots \\
L_{n}^{1} & \ldots & L_{n}^{n}
\end{array}\right)
$$

removing the $i$-th row. Since $\widetilde{\Omega}$ is a rational $n$-form on $\mathbb{P}^{n}$, following [9][Corollary 2.11] it can be written as $\frac{P \Psi}{Q^{n}}$, and we deduce that

$$
\frac{M_{i}}{(-1)^{i} \xi_{i}}=P
$$

for all $i=0, \ldots, n$. $P$ is a polynomial of degree $n-1$ and it corresponds to $\widetilde{\Omega}$ via the isomorphism $H^{0}\left(V, \Omega_{\mathbb{P}^{n} \mid V}^{n}(2 n)\right) \cong H^{0}\left(V, \mathcal{O}_{V}(n-1)\right)$. Hence by (3.1) we have that the form $\Omega \in H^{0}\left(V, \mathcal{O}_{V}(n+d-1)\right)$ given by $P R$ is a canonical choice of adjoint form for $W=\left\langle\eta_{1}, \ldots, \eta_{n}\right\rangle$ and $\xi$.

Remark 3.2 Alternatively this can be seen using the Euler sequence on $V$ :

$$
0 \rightarrow \mathcal{O}_{V} \rightarrow \bigoplus^{n+1} \mathcal{O}_{V}(1) \rightarrow \Theta_{\mathbb{P}^{n} \mid V} \rightarrow 0
$$

This sequence, dualized and conveniently tensorized gives

$$
0 \rightarrow \Omega_{\mathbb{P}^{n} \mid V}^{1}(2) \rightarrow \bigoplus_{i=1}^{n+1} \mathcal{O}_{V}(1) \rightarrow \mathcal{O}_{V}(2) \rightarrow 0 .
$$

The sections $\tilde{s}_{i}$ are associated via the first morphism to an $n+1$-uple of linear polynomials $\left(L_{i}^{0}, \ldots, L_{i}^{n}\right)$. Then, taking the wedge product of (3.16) we obtain an exact sequence

$$
0 \rightarrow \Omega_{\mathbb{P}^{n} \mid V}^{n}(2 n) \cong \mathcal{O}_{V}(n-1) \rightarrow \bigwedge^{n} \mathcal{O}_{V}(1)=\bigoplus^{n+1} \mathcal{O}_{V}(n) \rightarrow \Omega_{\mathbb{P}^{n} \mid V}^{n-1}(2 n) \rightarrow 0
$$


where the morphism $\mathcal{O}_{V}(n-1) \rightarrow \bigoplus^{n+1} \mathcal{O}_{V}(n)$ is given by

$$
G \mapsto\left(G \xi_{0}, \ldots,(-1)^{n} G \xi_{n}\right) .
$$

Since $\widetilde{\Omega}=\Lambda^{n}\left(\tilde{s_{1}} \wedge \ldots \wedge \tilde{s_{n}}\right) \in H^{0}\left(V, \Omega_{\mathbb{P}^{n} \mid V}^{n}(2 n)\right)$ is sent exactly to $\left(L_{0}^{0}, \ldots, L_{0}^{n}\right) \wedge \ldots \wedge$ $\left(L_{n}^{0}, \ldots, L_{n}^{n}\right)=\left(M_{0}, \ldots, M_{n}\right)$ (using the same notation as above), then we conclude that $\widetilde{\Omega}$ corresponds in $H^{0}\left(V, \mathcal{O}_{V}(n-1)\right)$ to a polynomial $P$ which satisfies

$$
\frac{M_{i}}{(-1)^{i} \xi_{i}}=P \text {. }
$$

\subsection{The adjoint sublinear systems obtained by meromorphic 1-forms}

To study the conditions given in (2.6) and (2.7), we need to describe the sections

$$
\widetilde{\Omega_{i}}:=\Lambda^{n-1}\left(\tilde{s_{1}} \wedge \ldots \wedge \hat{\tilde{s_{i}}} \wedge \ldots \wedge \tilde{s_{n}}\right) \in H^{0}\left(V, \Omega_{\mathbb{P}^{n} \mid V}^{n-1}(2 n-2)\right)
$$

(c.f. (2.4)) and their images in $H^{0}\left(V, \Omega_{V}^{n-1}(2(n-1))\right)=H^{0}\left(V, \mathcal{O}_{V}(n+d-3)\right)$ that we have denoted by $\omega_{i}$.

A computation similar to the above shows that

$$
\widetilde{\Omega_{i}}=\Lambda^{n-1}\left(\tilde{s_{1}} \wedge \ldots \wedge \hat{\tilde{s_{i}}} \wedge \ldots \wedge \tilde{s_{n}}\right)=\frac{1}{Q^{n-1}} \sum_{j<k} M_{j k}^{i} d \xi_{0} \wedge \ldots \wedge d \hat{\xi}_{j} \wedge \ldots \wedge d \hat{\xi}_{k} \wedge \ldots \wedge d \xi_{n}
$$

where $M_{j k}^{i}$ is the determinant of the matrix obtained by (3.13) removing the $i$-th column and the $j$-th and $k$-th rows. On the other hand, rearranging the expression of [9][Theorem 2.9] we can write

$$
\widetilde{\Omega_{i}}=\frac{1}{Q^{n-1}} \sum_{j} A_{j}^{i}\left(\sum_{k \neq j}(-1)^{k+j} \operatorname{sgn}(k-j) \xi_{k} d \xi_{0} \wedge \ldots \wedge d \hat{\xi}_{j} \wedge \ldots \wedge d \hat{\xi}_{k} \wedge \ldots \wedge d \xi_{n}\right)
$$

with $\operatorname{deg} A_{j}^{i}=n-2$.

Comparing (3.20) and (3.21) gives

$$
M_{j k}^{i}=(-1)^{j+k}\left(A_{j}^{i} \xi_{k}-\xi_{j} A_{k}^{i}\right) .
$$

As before this can be computed also via the Euler sequence.

We call $\Xi_{j}:=\sum_{k \neq j}(-1)^{k+j} \operatorname{sgn}(k-j) \xi_{k} d \xi_{0} \wedge \ldots \wedge d \hat{\xi}_{j} \wedge \ldots \wedge d \hat{\xi}_{k} \wedge \ldots \wedge d \xi_{n}$. Note that the sections $\Xi_{j}$, for $j=0, \ldots, n$ give a basis of $H^{0}\left(V, \Omega_{\mathbb{P}^{n} \mid V}^{n-1}(n)\right)$.

Proposition $3.3 \omega_{i}=\sum_{j} A_{j}^{i} \cdot F_{j}$ in $H^{0}\left(V, \mathcal{O}_{V}(n+d-3)\right)$

Proof It is enough to show that the image of $\Xi_{j}$ through the morphism $\Omega_{\mathbb{P}^{n} \mid V}^{n-1}(n) \rightarrow \mathcal{O}_{V}$ $(d-1)$ is $F_{j}$. Consider the exact sequence of the tangent sheaf of $V$ :

$$
0 \rightarrow \Theta_{V} \rightarrow \Theta_{\mathbb{P}^{n} \mid V} \rightarrow \mathcal{O}_{V}(d) \rightarrow 0 .
$$

The beginning of the Koszul complex is

$$
\bigwedge^{n} \Theta_{\mathbb{P}^{n} \mid V} \otimes \mathcal{O}_{V}(-d) \rightarrow \bigwedge^{n-1} \Theta_{\mathbb{P}^{n} \mid V}
$$

which, tensored by $\mathcal{O}_{V}(-n)$, gives

$$
\bigwedge^{n} \Theta_{\mathbb{P}^{n} \mid V} \otimes \mathcal{O}_{V}(-n-d) \rightarrow \bigwedge^{n-1} \Theta_{\mathbb{P}^{n} \mid V} \otimes \mathcal{O}_{V}(-n) .
$$


This is exactly the dual of $\Omega_{\mathbb{P}^{n} \mid V}^{n-1}(n) \rightarrow \mathcal{O}_{V}(d-1)$. Hence we only need to show that the morphism (3.25) composed with the contraction by $\Xi_{i}$

$$
\bigwedge^{n-1} \Theta_{\mathbb{P}^{n} \mid V} \otimes \mathcal{O}_{V}(-n) \stackrel{\Xi_{i}}{\rightarrow} \mathcal{O}_{V}
$$

is the multiplication by $F_{i}$. This is easy to see by a standard local computation.

Remark 3.4 We immediately have that the polynomials associated to the sections $\omega_{i}$ are in the Jacobian ideal of $V$.

Condition (2.7), that is

$$
\Omega \in \operatorname{Im}\left(H^{0}\left(V, \mathcal{O}_{V}(2)\right) \otimes \lambda^{n} W \rightarrow H^{0}\left(V, \mathcal{O}_{V}(n+d-1)\right)\right),
$$

can be written, modulo $F$, as

$$
R P=\sum \omega_{i} \cdot S_{i}=\sum_{i, j} A_{j}^{i} \cdot F_{j} \cdot S_{i},
$$

where $\operatorname{deg} S_{i}=2$. In particular this implies that $R P$ is in the Jacobian ideal of $V$.

Proposition 3.5 The base locus $D_{W}$ of the linear system $\left|\lambda^{n} W\right|$ is zero for the generic $W$.

Proof By [11][Proposition 3.1.6] it is enough to prove that $H^{0}\left(V, \Omega_{V}^{1}(2)\right)$ generically generates the sheaf $\Omega_{V}^{1}(2)$ and that $D_{H^{0}\left(V, \Omega_{V}^{1}(2)\right)}=0$. We have an explicit basis for $H^{0}\left(V, \Omega_{V}^{1}(2)\right)$ given by

$$
\frac{\xi_{i} d \xi_{j}-\xi_{j} d \xi_{i}}{Q}
$$

where $i<j$ and $\operatorname{deg} Q=2$. The vector space $\lambda^{n} H^{0}\left(V, \Omega_{V}^{1}(2)\right) \subset H^{0}\left(V, \mathcal{O}_{V}(n+d-3)\right)$ is obviously nonzero, hence $H^{0}\left(V, \Omega_{V}^{1}(2)\right)$ generically generates the sheaf $\Omega_{V}^{1}(2)$.

It remains to prove that $D_{H^{0}\left(V, \Omega_{V}^{1}(2)\right)}=0$. An easy computation (for example by induction) shows that $\lambda^{n} H^{0}\left(V, \Omega_{V}^{1}(2)\right)$ contains all the polynomials of the form

$$
\xi_{i_{1}} \xi_{i_{2}} \ldots \xi_{i_{n-2}} \frac{\partial F}{\partial \xi_{j}}
$$

where $\left\{i_{1}, \ldots, i_{n-2}\right\} \subset\{1, \ldots, n+1\}$ and $j \notin\left\{i_{1}, \ldots, i_{n-2}\right\}$. Since $V$ is smooth, these polynomials do not vanish simultaneously on a divisor, hence $D_{H^{0}\left(V, \Omega_{V}^{1}(2)\right)}=0$, and we are done.

\subsection{On Griffiths's proof of infinitesimal Torelli Theorem}

In this section we will prove Theorem [C] of the Introduction.

It is well known by [9] that the deformation $\xi$ is trivial if and only if $R$ lies in the Jacobian ideal $\mathcal{J}$ of the variety $V$. The following lemma gives a translation of this condition in the setting of adjoint forms.

Lemma 3.6 $R$ is in the Jacobian ideal $\mathcal{J}$ if and only if $\Omega \in \operatorname{Im}\left(H^{0}\left(V, \mathcal{O}_{V}(2)\right) \otimes \lambda^{n} W \rightarrow\right.$ $\left.H^{0}\left(V, \mathcal{O}_{V}(n+d-1)\right)\right)$ for the generic $\Omega$.

Proof If $\Omega \in \operatorname{Im}\left(H^{0}\left(V, \mathcal{O}_{V}(2)\right) \otimes \lambda^{n} W \rightarrow H^{0}\left(V, \mathcal{O}_{V}(n+d-1)\right)\right)$, then by the Adjoint Theorem, $\xi_{D_{W}}=0$. Since $D_{W}=0$, the deformation is trivial, hence $R$ lies in the Jacobian ideal.

Viceversa if $R \in \mathcal{J}$, the deformation is trivial and by theorem (2.9), we have that $\Omega \in$ $\operatorname{Im}\left(H^{0}\left(V, \mathcal{O}_{V}(2)\right) \otimes \lambda^{n} W \rightarrow H^{0}\left(V, \mathcal{O}_{V}(n+d-1)\right)\right)$. 
Our theory gives another characterization for $[R] \in\left(\mathbb{C}\left[\xi_{0}, \ldots, \xi_{n}\right] / \mathcal{J}\right)_{d} \simeq H^{1}\left(V, \Theta_{V}\right)$ to be trivial.

Proposition 3.7 Assume that $\operatorname{deg} R=d>3$. Then $R$ is in the Jacobian ideal $\mathcal{J}$ if and only if $R P \in \mathcal{J}$ for every polynomial $P \in H^{0}\left(V, \mathcal{O}_{V}(n-1)\right)$ corresponding to a generalized adjoint $\widetilde{\Omega} \in H^{0}\left(V, \Omega_{\mathbb{P}^{n} \mid V}^{n}(2 n)\right)$.

Proof One implication is trivial.

To prove the other one the idea is to show that every monomial of $H^{0}\left(V, \mathcal{O}_{V}(n-1)\right)$ corresponds to a suitable generalized adjoint. Hence, if $R P \in \mathcal{J}$ for every polynomial $P \in$ $H^{0}\left(V, \mathcal{O}_{V}(n-1)\right)$ corresponding to a generalized adjoint, we have that $R \cdot H^{0}\left(V, \mathcal{O}_{V}(n-\right.$ 1)) $\subset \mathcal{J}$ and we are done by Macaulay Theorem (c.f. [16] Theorem 6.19 and Corollary 6.20).

We work by induction at the level of $\mathbb{P}^{n}$, since $H^{0}\left(\mathbb{P}^{n}, \mathcal{O}_{\mathbb{P}}(n-1)\right) \rightarrow H^{0}\left(V, \mathcal{O}_{V}(n-1)\right)$ is surjective. The base of the induction is for $n=2$. A simple computation shows that the map

$$
\bigwedge^{2} H^{0}\left(\mathbb{P}^{2}, \Omega_{\mathbb{P}^{2}}^{1}(2)\right) \rightarrow H^{0}\left(\mathbb{P}^{2}, \mathcal{O}_{\mathbb{P}^{2}}(1)\right)
$$

is surjective because its image contains the canonical basis of degree one monomials.

For the general case we show that every monomial of degree $n-1$ is given by a generalized adjoint. Consider the natural homomorphism:

$$
\bigwedge^{n} H^{0}\left(\mathbb{P}^{n}, \Omega_{\mathbb{P}^{n}}^{1}(2)\right) \rightarrow H^{0}\left(\mathbb{P}^{n}, \mathcal{O}_{\mathbb{P}^{n}}(n-1)\right)
$$

and take a monomial $M$ with $\operatorname{deg} M=n-1$. There is a variable $\xi_{i}$ which does not appear in $M$. We restrict to the hyperplane $\xi_{i}=0$ and we use induction on $\frac{M}{\xi_{j}}$, where $\xi_{j}$ appears in $M$. There exist $s_{1}, \ldots, s_{n-1} \in H^{0}\left(\mathbb{P}^{n-1}, \Omega_{\mathbb{P}^{n-1}}^{1}(2)\right)$ with $s_{1} \wedge \ldots \wedge s_{n-1}$ which corresponds to $\frac{M}{\xi_{j}}$, that is

$$
s_{1} \wedge \ldots \wedge s_{n-1}=\frac{M \Psi^{\prime}}{\xi_{j} \cdot Q^{n-1}}
$$

where $\Psi^{\prime}=\sum_{k=0, k \neq i}^{n}(-1)^{k} \xi_{k}\left(d \xi_{0} \wedge \ldots \wedge \hat{d \xi_{i}} \ldots \wedge d \hat{\xi}_{k} \ldots \wedge d \xi_{n}\right)$ gives a basis of $H^{0}\left(\mathbb{P}^{n-1}, \Omega_{\mathbb{P}^{n-1}}^{n-1}(n)\right)$. It is easy to see that

$$
s_{1} \wedge \ldots \wedge s_{n-1} \wedge \frac{\left(\xi_{j} d \xi_{i}-\xi_{i} d \xi_{j}\right)}{Q}=\frac{M \Psi}{Q^{n}},
$$

i.e. $M$ corresponds to a generalized adjoint, which is exactly our thesis.

From the previous results we deduce immediately Theorem [C] of the Introduction.

Acknowledgements This research is supported by MIUR funds, PRIN project Geometria delle varietà algebriche (2010), coordinator A. Verra. The first author is also supported by funds of the Università degli Studi di Udine-Finanziamento di Ateneo per progetti di ricerca scientifica.

\section{References}

1. Á. Barja, M., González-Alonso, V., Naranjo, J.C.: Xiao’s conjecture for general fibred surfaces, arXiv:1401.7502, (2014)

2. Collino, A., Naranjo, J.C., Pirola, G.P.: The Fano normal function. J. Math. Pures Appl.(9) 98(3), 346-366 (2012) 
3. Collino, A., Pirola, G.P.: The Griffiths infinitesimal invariant for a curve in its Jacobian. Duke Math. J. 78(1), 59-88 (1995)

4. Donagi, R.: Generic Torelli for projective hypersurfaces. Compositio Math. 50(2-3), 325-353 (1983)

5. González-Alonso, V.: Hodge numbers of irregular varieties and fibrations, Ph.D. Thesis, (2013)

6. González-Alonso, V.: On deformations of curves supported on rigid divisors. Ann. Mat. Pura Appl. (4) 195(1), 111-132 (2016)

7. Green, M.L.: The period map for hypersurface sections of high degree of an arbitrary variety. Compositio Math. 55, 135-156 (1985)

8. Green, M.L.: Infinitesimal Methods in Hodge theory. Springer, Berlin (1994). CIME Notes

9. Griffiths, P.: On the periods of certain rational integrals: I,II, Ann. Math. (2) 90 (1969), 460-495; ibid. (2) 90 (1969) 496-541

10. Pirola, G.P., Rizzi, C.: Infinitesimal invariant and vector bundles. Nagoya Math. J. 186, 95-118 (2007)

11. Pirola, G.P., Zucconi, F.: Variations of the Albanese morphisms. J. Algebraic Geom. 12(3), 535-572 (2003)

12. Raviolo, E.: Some geometric applications of the theory of variations of Hodge structures, Ph.D. Thesis

13. Rizzi, L., Zucconi, F.: Differential forms and quadrics of the canonical image, arXiv:1409.1826, 23 pages

14. Rizzi, L., Zucconi, F.: On Green's proof of infinitesimal Torelli theorem for hypersurfaces, Preprint

15. Voisin, C.: Hodge theory and complex algebraic geometry, I. Translated from the French by Leila Schneps. Cambridge Studies in Advanced Mathematics, 76. Cambridge University Press, Cambridge, (2002)

16. Voisin, C.: Hodge theory and complex algebraic geometry, II. Translated from the French by Leila Schneps. Cambridge Studies in Advanced Mathematics, 77. Cambridge University Press, Cambridge, (2003) 